\title{
Beryllium abundances in stars with planets
}

\section{Extending the sample ${ }^{\star}$}

\author{
M. C. Gálvez-Ortiz ${ }^{1}$, E. Delgado-Mena², J. I. González Hernández ${ }^{2,3}$, G. Israelian² , N. C. Santos ${ }^{4}$, \\ R. Rebolo ${ }^{2,5}$, and A. Ecuvillon ${ }^{2}$ \\ ${ }^{1}$ Centre for Astrophysics Research, Science and Technology Research Institute, University of Hertfordshire, \\ Hatfield AL10 9AB, UK \\ e-mail: M.Galvez-Ortiz@herts.ac.uk \\ 2 Instituto de Astrofísica de Canarias, 38200 La Laguna, Tenerife, Spain \\ 3 Dpto. de Astrofísica y Ciencias de la Atmósfera, Facultad de Ciencias Físicas, Universidad Complutense de Madrid, \\ 28040 Madrid, Spain \\ ${ }^{4}$ Centro de Astrofísica, Universidade do Porto, Rua das Estrelas, 4150-762 Porto, Portugal \\ ${ }^{5}$ Consejo Superior de Investigaciones Científicas, Spain
}

Received 8 December 2009 / Accepted 31 March 2011

\section{ABSTRACT}

\begin{abstract}
Context. Chemical abundances of light elements such as beryllium in planet-host stars allow us to study the planet formation scenarios and/or investigate possible surface pollution processes.

Aims. We present here an extension of previous beryllium abundance studies. The complete sample consists of 70 stars that host planets and 30 stars without known planetary companions. The aim of this paper is to further assess the trends found in previous studies with fewer objects. This will provide more information on the processes of depletion and mixing of light elements in the interior of late-type stars, and will provide possible explanations for the abundance differences between stars that host planets and "single" stars.

Methods. Using high-resolution UVES spectra, we measure beryllium abundances of 26 stars that host planets and one "single" star mainly using the $\lambda 3131.065 \AA \mathrm{Be}$ II line, by fitting synthetic spectra to the observational data. We also compile beryllium abundance measurements of 44 stars hosting planets and 29 "single" stars from the literature, resulting in a final sample of 100 objects.

Results. We confirm that the beryllium content is roughly the same in stars hosting planets and in "single" stars at temperatures $T_{\text {eff }} \gtrsim 5700 \mathrm{~K}$. The sample is still small for $T_{\text {eff }} \lesssim 5500 \mathrm{~K}$, but it seems that the scatter in Be abundances of dwarf stars is slightly higher at these cooler temperatures.

Conclusions. We search for distinctive characteristics of planet hosts through correlations of Be abundance versus Li abundance, age, metallicity, and oxygen abundance. These could provide some insight into the formation and evolution of planetary systems, but we did not find any clear correlation.
\end{abstract}

Key words. stars: abundances - stars: fundamental parameters - planetary systems - planets and satellites: formation stars: atmospheres

\section{Introduction}

Since the discovery of the first extrasolar planets, many efforts have been made to characterise planet-host stars and to find the features that can distinguish them from stars that do not have any known planetary companion (e.g. Santos et al. 2004a; Fischer $\&$ Valenti 2005). The study of chemical composition and abundances in general (e.g. Ecuvillon et al. 2004a,b, 2006; Gilli et al. 2006; Neves et al. 2009), and, in particular, of the light elements Li, Be, and B (e.g. Santos et al. 2002, 2004c; Israelian et al. 2004, 2009), provided some hints on the influence of a planetary companion in the composition and evolution of stars. These light elements are destroyed by ( $\mathrm{p}, \alpha)$-reactions at relatively low temperatures (about 2.5, 3.5 and $5.0 \times 10^{6} \mathrm{~K}$ for $\mathrm{Li}, \mathrm{Be}$, and $\mathrm{B}$, respectively). Thus, light elements and their abundance ratios are good tracers of the stellar internal structure and allow us to extract valuable information about the mixing mechanism and rotation behaviour in these stars (e.g. Pinsonneault et al. 1990).

* Based on observations obtained with UVES at VLT Kueyen $8.2 \mathrm{~m}$ telescope in programme 074.C-0134(A).
Several groups (e.g. Gonzalez et al. 2001; Santos et al. 2001, 2004a) have found a correlation between stellar metallicity and the presence of a giant planetary companion among solar-like stars. It has been also extensively discussed whether the trend is due to a "primordial" origin, i.e. that the frequency of a planetary companion is a function of the proto-planetary disc's metal composition (see e.g. Santos et al. 2004a); or if the trend is due to a posterior "pollution", i.e. that the metallicity excess is caused by matter accretion after reaching the main sequence (see e.g. Pasquini et al. 2007). Most studies suggest the "primordial" scenario, although some examples may indicate that both cases occur (Israelian et al. 2001, 2003; Ashwell et al. 2005; Laws et al. 2003).

However, it is not clear whether a direct relationship between metallicity and probability of planet formation exists or if it is only applicable to the "type" of planets that have been discovered so far. Santos et al. (2004a) suggested that two distinct populations of exoplanets can be present depending on whether or not the planet is formed by a metallicity-dependent process. Recently, Haywood (2009) has also proposed that the metallicity excess in the sample of stars hosting planets may 
come from a dynamical effect of galactic nature (i.e. migration of stars in the galactic disc), and may not be related with the giant planets' formation process.

Israelian et al. (2004) found that exoplanet hosts are significantly more Li-depleted than comparison stars but only in the range $5600-5850 \mathrm{~K}$. This result has been confirmed by Chen \& Zhao (2006), Takeda et al. (2007), and Gonzalez (2008). On the other hand, Gonzalez (2008) proposed that planet-host stars hotter than $5900 \mathrm{~K}$ have more Li than the comparison ones. There is observational evidence that $\mathrm{Li}$ depletion is connected with the rotational history of the star (e.g. García López 1994; Randich et al. 1997). Thus, similar stars, i.e. those with similar stellar parameters and age, should have different depletion rates depending on the proto-planetary disc mass and composition and its effect on the rotation of the parent star (Bouvier 2008).

More recently, Israelian et al. (2009) has confirmed that Li is more depleted in Sun-like stars that host planets than in similar stars without detected planets. These stars with and without planets, which fall in the effective temperature range $5700-5850 \mathrm{~K}$, do not show any differences in the correlation patterns of their Li abundances with age and metallicity, which points to a connection between the presence of planets and the low Li abundance measured in Sun-like planet-host stars.

Whatever causes the metallicity "excess" in planet-host stars, light elements can still provide insights on the influence of planets on the parent-star evolution and in the internal evolution of stars in general, they serve as a key for understanding pollution "events", and also as tracers of the possible planet-disc interaction.

In previous works directly related with this paper (Santos et al. 2002, 2004b,c), a comparison between stars that host planets and stars without known planets revealed that, with some exceptions, the two samples follow approximately the same behaviour in the variation of Be abundance with the temperature, similar to the $\mathrm{Li}$ trend. This behaviour shows a Be abundance maximum near $6100 \mathrm{~K}$, decreasing towards higher and lower temperatures and a Be "gap" for solar-temperature stars. Santos et al. (2004c) also remarked the possible tendency of the planet hosts to be more Be-rich, at least for $T_{\text {eff }} \gtrsim 5700 \mathrm{~K}$.

In this paper we derive beryllium abundances for 26 stars with planets and one star without planets, and collect the lithium abundances for most of them. This sample enlarges the previous samples of 44 stars with planets and 29 stars without known planetary companions reported in García López \& Pérez de Taoro (1998); Santos et al. (2004c). Thus, we end up with a sample of roughly 100 stars of which 70 host planets. We studied the behaviour of these abundances with the spectral type and luminosity class in both samples of stars in order to further assess the trends found before, and to study possible interactions between extrasolar planets and their parent stars and its connection to the light-element abundances through time.

\section{Observations and data reduction}

We obtained near-UV high-resolution spectra of the targets using the UVES spectrograph at the 8.2-m Kueyen VLT (UT2) telescope (run ID 074.C-0134(A)) on 21 and 22 December 2004 in the blue arm with a wavelengh coverage of $\lambda \lambda 3025-3880 \AA$. These spectra have a spectral resolution $\lambda / \delta \lambda \sim 70000$.

All data were reduced using $\operatorname{IRAF}^{1}$ tools in the echelle package. Standard background correction, flat-field, and extraction

\footnotetext{
1 IRAF is distributed by the National Optical Observatory, which is operated by the Association of Universities for Research in Astronomy, Inc., under contract with the National Science Foundation.
}

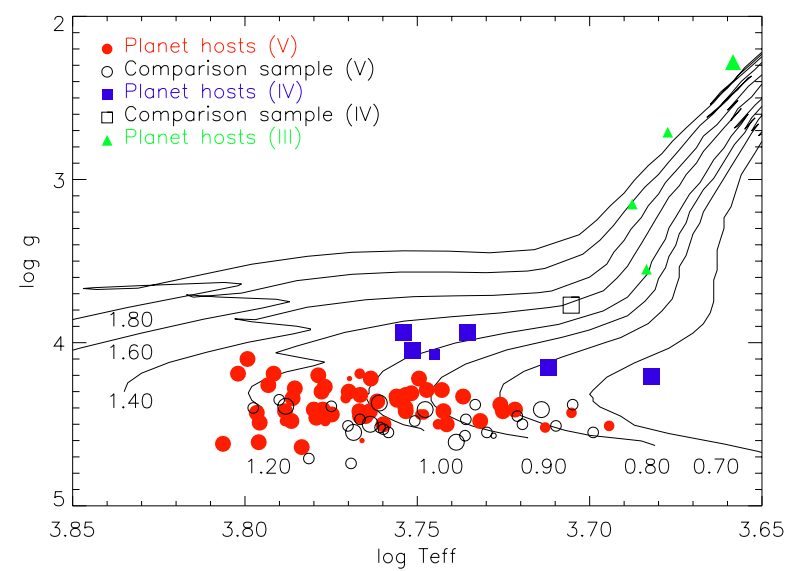

Fig. 1. HR diagram: surface gravity vs effective temperatures of the stars in our sample including the stars in Tables 1-3. We overplot solar metallicity tracks from Girardi et al. (2000) for 0.7 to $1.8 M_{\odot}$. The luminosity classes of the stars in the sample are assigned according to each stars position in this diagram (see Sect. 3). Metallicity is represent by the size of the symbol.

procedures were used. The wavelength calibration was done using a ThAr lamp spectrum taken during the same night. Finally, we normalized the spectra by a low-order polynomial fit to the observed continuum.

\section{Sample}

The total sample of $\sim 100$ stars, including 70 planet-host stars, covers a wide temperature range from 4500 to $6400 \mathrm{~K}$. Thus, the stars hosting planets have spectral types between F6 to K2 and three luminosity classes (V, IV and III). The luminosity classes were assigned following a criterion based on the position of the star in the Hertzsprung-Russell (HR) diagram, and also on their surface gravity, i.e. dwarfs (V) have typically $\log g>4$, subgiants (IV) have $3.3<\log g<4$ and giants (III) have $\log g<3.3$. We built an effective temperature-surface gravity plot with solar metallicity tracks from Girardi et al. (2000) for 0.7 to $1.8 M_{\odot}$, and according to each star position in this diagram (see Fig. 1), we assigned a luminosity class, which we present in Col. 10 of Table 1 and Col. 7 of Tables 2 and 3. In Fig. 1 we represent the metallicity of the objects by increasing the symbol size according to three possible metallicity ranges from smaller to bigger size: $[\mathrm{Fe} / \mathrm{H}]<-0.5,-0.5<[\mathrm{Fe} / \mathrm{H}]<0.0$ and $0.0<[\mathrm{Fe} / \mathrm{H}]<0.5$, respectively. Thus, our sample of planethost stars with new Be abundance measurements contains 3 giants, 2 subgiants and 21 dwarfs that give a total of 4 giants, 6 subgiants and 60 dwarfs in this study. The 30 stars without known planets of our sample cover the same spectral types but only two of them are a subgiant star. All new objects are collected in Table 1 (with 26 stars with planets and one star without known planetary companions). Stars with and without known planets from the literature are listed in Tables 2 and 3, respectively.

\section{Stellar parameters}

Stellar parameters were taken from the detailed analysis of Sousa et al. (2008) when available, otherwise from Santos et al. (2004a, 2005). These parameters are determined from ionization and excitation equilibrium of $\mathrm{Fe}_{\mathrm{I}}$ and Fe II. They used the 2002 version of the code MOOG $^{2}$ (Sneden 1973) and a grid of

\footnotetext{
2 The source code of MOOG 2002 can be downloaded at http://verdi.as.utexas. edu/moog.html
} 
Table 1. Stellar parameters and Be abundances measured in this work.

\begin{tabular}{|c|c|c|c|c|c|c|c|c|c|c|c|c|c|}
\hline Name & $\begin{array}{l}T_{\text {eff }} \\
{[\mathrm{K}]}\end{array}$ & $\begin{array}{c}\log g \\
{\left[\mathrm{~cm} \mathrm{~s}^{-2}\right]}\end{array}$ & $\begin{array}{c}\xi_{\mathrm{t}} \\
\mathrm{km} \mathrm{s}^{-1}\end{array}$ & {$[\mathrm{Fe} / \mathrm{H}]$} & $\begin{array}{c}\text { Mass } \\
M_{\odot}\end{array}$ & $\begin{array}{c}V_{\text {sini }} \\
\mathrm{km} \mathrm{s}^{-1}\end{array}$ & $V$ & $\mathrm{SpT}^{d}$ & $\mathrm{LC}^{e}$ & $\begin{array}{c}\log N(\mathrm{Be}) \\
{[\mathrm{dex}]}\end{array}$ & $\begin{array}{c}\log N(\mathrm{Li}) \\
{[\mathrm{dex}]}\end{array}$ & $\begin{array}{r}{[\mathrm{O} / \mathrm{H}]^{f}} \\
{[\mathrm{dex}]}\end{array}$ & $\begin{array}{r}\mathrm{Age}^{g} \\
\text { Gyr }\end{array}$ \\
\hline HD $142^{a}$ & 6403 & 4.62 & 1.74 & 0.09 & 1.29 & 11.3 & 5.70 & F7V & $\overline{\mathrm{V}}$ & 1.31 & 3.00 & $0.07 \pm 0.13$ & 3.6 \\
\hline $\mathrm{HD} 1237^{a}$ & 5514 & 4.50 & 1.09 & 0.07 & 0.94 & 5.5 & 6.59 & G8.5Vk: & V & 1.14 & 2.19 & $0.07 \pm 0.10$ & 0.15 \\
\hline HD $4208^{a}$ & 5599 & 4.44 & 0.78 & -0.28 & 0.83 & 2.8 & 7.79 & G7VFe-1H-05 & V & 0.94 & $<0.20$ & $-0.14 \pm 0.08$ & 4.47 \\
\hline HD $23079^{a}$ & 5980 & 4.48 & 1.12 & -0.12 & 1.01 & 3.4 & 7.12 & F9.5V & $\mathrm{V}$ & 1.10 & 2.21 & $0.02 \pm 0.09$ & 8.4 \\
\hline HD $28185^{a}$ & 5667 & 4.42 & 0.94 & 0.21 & 0.98 & 2.5 & 7.80 & G6.5IV-V & V & 0.98 & $<0.26$ & $0.23 \pm 0.10$ & 12.2 \\
\hline HD $30177^{b}$ & 5588 & 4.29 & 1.08 & 0.39 & 1.01 & $2.96^{1}$ & 8.41 & G8V & V & 0.96 & $<0.35^{2}$ & $0.31 \pm 0.12$ & 8.30 \\
\hline HD $33636^{b *}$ & 6046 & 4.71 & 1.79 & -0.08 & 1.16 & $3.08^{1}$ & 7.06 & G0VH-03 & V & 1.34 & 2.64 & $0.02 \pm 0.11$ & 8.1 \\
\hline HD $37124^{b}$ & 5546 & 4.50 & 0.80 & -0.38 & 0.75 & 1.22 & 7.68 & G4IV-V & V & 0.94 & $<0.31$ & $-0.06 \pm 0.08$ & 0.57 \\
\hline HD $39091^{a}$ & 6003 & 4.42 & 1.12 & 0.09 & 1.10 & 3.3 & 5.67 & G0V & V & 1.11 & 2.39 & $0.23 \pm 0.08$ & 6.0 \\
\hline HD $47536^{b}$ & 4554 & 2.28 & 1.82 & -0.54 & - & $1.93^{3}$ & 5.26 & KOIII & III & $<-1.32$ & $<-0.46^{3}$ & $-0.29 \pm 0.12$ & - \\
\hline HD $50554^{b}$ & 6026 & 4.41 & 1.11 & 0.01 & 1.09 & $3.88^{1}$ & 6.84 & F8V & $\mathrm{V}$ & 1.18 & 2.57 & $0.17 \pm 0.09$ & 7.0 \\
\hline HD $59686^{c}$ & 4871 & 3.15 & 1.85 & 0.28 & - & $0.96^{3}$ & 5.45 & K2III & III & $<-0.75$ & $<0.07^{3}$ & $0.14 \pm 0.16$ & - \\
\hline HD $65216^{a}$ & 5612 & 4.44 & 0.78 & -0.17 & 0.87 & 2.3 & 7.97 & G5V & $\mathrm{V}$ & 1.05 & 1.28 & $0.00 \pm 0.08$ & - \\
\hline HD $70642^{a}$ & 5668 & 4.40 & 0.82 & 0.18 & 0.98 & 2.8 & 7.18 & G6VCN+05 & V & 0.90 & $<0.46$ & $0.17 \pm 0.09$ & 10.2 \\
\hline HD $72659^{b}$ & 5995 & 4.30 & 1.42 & 0.03 & 1.16 & $2.21^{1}$ & 7.46 & G0V & V & 1.25 & 2.38 & $0.21 \pm 0.09$ & 8.2 \\
\hline HD $73256^{a}$ & 5526 & 4.42 & 1.11 & 0.23 & 0.97 & 3.1 & 8.08 & G8IV-VFe+05 & V & 0.75 & $<0.54$ & $0.11 \pm 0.11$ & 15.9 \\
\hline HD $74156^{b}$ & 6112 & 4.34 & 1.38 & 0.16 & 1.27 & 4.32 & 7.61 & G0 & V & 1.33 & 2.75 & $0.32 \pm 0.08$ & 3.2 \\
\hline HD $88133^{c}$ & 5438 & 3.94 & 1.16 & 0.33 & 0.98 & $2.17^{1}$ & 8.06 & G5IV & IV & 0.65 & $1.91^{3}$ & $0.22 \pm 0.09$ & 9.56 \\
\hline HD $99492^{c}$ & 4810 & 4.21 & 0.72 & 0.26 & - & $1.36^{1}$ & 7.57 & $\mathrm{~K} 2 \mathrm{~V}$ & IV & $<-0.22$ & $<-0.01$ & $0.01 \pm 0.08$ & 4.49 \\
\hline HD $106252^{b}$ & 5899 & 4.34 & 1.08 & -0.01 & 1.02 & $1.93^{1}$ & 7.36 & G0V & V & 0.95 & 1.66 & $0.09 \pm 0.07$ & 9.2 \\
\hline HD $114729^{a}$ & 5844 & 4.19 & 1.23 & -0.28 & 0.94 & 2.3 & 6.69 & G0V & V & 0.99 & 2.00 & $-0.01 \pm 0.07$ & 11.9 \\
\hline HD $117207^{a}$ & 5667 & 4.32 & 1.01 & 0.22 & 0.98 & 1.8 & 7.26 & G7IV-V & V & 0.94 & $<0.12$ & $0.19 \pm 0.09$ & 16.1 \\
\hline HD $117618^{a}$ & 5990 & 4.41 & 1.13 & 0.03 & 1.08 & 3.2 & 7.17 & G0V & V & 1.10 & 2.29 & $0.14 \pm 0.09$ & 6.72 \\
\hline HD $213240^{a}$ & 5982 & 4.27 & 1.25 & 0.14 & 1.21 & 4.0 & 6.80 & G0/G1V & V & 1.41 & 2.54 & $0.25 \pm 0.08$ & 3.60 \\
\hline HD $216435^{a}$ & 6008 & 4.20 & 1.34 & 0.24 & 1.33 & 5.9 & 6.03 & G0V & V & 1.26 & 2.77 & $0.23 \pm 0.12$ & 5.40 \\
\hline HD $216437^{b}$ & 5887 & 4.30 & 1.31 & 0.25 & 1.20 & 2.6 & 6.06 & $\mathrm{G} 1 \mathrm{VFe}+03$ & $\mathrm{~V}$ & 1.32 & 1.96 & $0.22 \pm 0.10$ & 8.7 \\
\hline HD $219449^{c}$ & 4757 & 2.71 & 1.71 & 0.05 & - & 5.10 & 4.21 & KOIII & III & $<-1.1$ & $<-0.16^{3}$ & $-0.10 \pm 0.13$ & - \\
\hline
\end{tabular}

Notes. We measured Li abundances for four stars. Li abundances without a label were taken from Israelian et al. (2009). ${ }^{(a)}$ Values taken from Sousa et al. (2008). ${ }^{(b)}$ Values taken from Santos et al. (2004a). ${ }^{(c)}$ Values taken from Santos et al. (2005). ${ }^{(d)}$ Values taken from SIMBAD. ${ }^{(e)}$ Luminosity class assigned by comparing the stellar parameters with isochrones from Girardi et al. (2000). ( $f$ ) Values taken from Ecuvillon et al. (2006). (g) Values taken from Saffe et al. (2005). ${ }^{(1)}$ Data taken from Fischer \& Valenti (2005). (2) Data taken from Israelian et al. (2004). ${ }^{(3)}$ Data measured in this work. $\left.{ }^{*}\right)$ Stars without planet companion.

local thermodynamical equilibrium (LTE) model atmospheres (Kurucz 1993). The adopted parameters, effective temperature, $T_{\text {eff }}$, surface gravity, $\log g$, metallicities, $[\mathrm{Fe} / \mathrm{H}]$, and masses are listed in Table 1. The mean values of the uncertainties on the parameters from Santos et al. (2004a, 2005) are of the order of $44 \mathrm{~K}$ for $T_{\mathrm{eff}}, 0.11 \mathrm{dex}$ for $\log g, 0.08 \mathrm{~km} \mathrm{~s}^{-1}$ for $\xi_{\mathrm{t}}, 0.06 \mathrm{dex}$ for metallicity and the adopted typical relative error for the masses is $0.05 M_{\odot}$, whereas from Sousa et al. (2008) these mean uncertainties are $25 \mathrm{~K}$ for $T_{\text {eff }}, 0.04$ dex for $\log g, 0.03 \mathrm{~km} \mathrm{~s}^{-1}$ for $\xi_{\mathrm{t}}$ and 0.02 dex for metallicity and $0.10 M_{\odot}$ for the masses. We refer to Sousa et al. (2008) and Santos et al. (2004a, 2005) for further details. In Tables 2 and 3 we give the main data of the sample of stars with and without planets from Santos et al. (2004b,c). We note here the uniformity of the adopted stellar parameters (see Sect. 5 in Sousa et al. 2008).

One should note that stellar masses are estimated from theoretical isochrones, which are strongly sensitive to the adopted helium and metal content of the star. In addition, the stellar mass, although it is a fundamental parameter, is relatively more uncertain than other stellar parameters, because it depends on the effective temperature, surface gravity, and metallicity of the star. However, unlike the stellar age, the stellar mass for mainsequence stars is typically better constrained from theoretical isochrones than for subgiant and/or giant stars (see e.g. Allende Prieto et al. 2004).

\section{Beryllium and lithium abundances}

The Be abundances were derived by fitting synthetic spectra to the data. These synthetic spectra were convolved with a Gaussian smoothing profile and a radial-tangential profile to take into account the spectral resolution and the macroturbulence, respectively. The latter was varied between 1.0 and $5.0 \mathrm{~km} \mathrm{~s}^{-1}$ and between K and F dwarfs (Gray 1992), respectively. A rotational profile was also added to account for the projected rotational velocity, $v \sin i$, of the sample stars, which is given in Table 1. These were calculated from the width of the CORALIE cross-correlation function (see Appendix of Santos et al. 2002), or taken from the literature (mostly Fischer \& Valenti 2005). A limb-darkening coefficient of 0.6 was adopted in all cases, and the overall metallicity was scaled to the iron abundance.

To derive the $\mathrm{Be}$ abundances, we fitted the entire observed spectral range between 3129.5 and $3132 \AA$, where two Be II lines are located ( $\lambda 3130.420$ and $\lambda 3131.065 \AA$ ). We mainly used the Be II $\lambda 3131.065 \AA$ line as the feature for deriving the best-fit Be abundance, and Be II $\lambda 3130.420 \AA$ was used only for checking, since it is highly blended with other elements lines.

We adopted the same line list as in Santos et al. $(2004 c)$, which provides a solar photospheric Be abundance of $\log N(\mathrm{Be})=1.1 \mathrm{dex}^{3}$, using the same models and tools as in this work. The main source of error is probably the placement of the continuum. An uncertainty on the continuum position of $3 \%$ yields to an error in the Be abundance of $\sim 0.05$ dex for solar-type stars (see e.g. Randich et al. 2002). We estimate this uncertainty to be $\sim 0.06$ dex at $T_{\text {eff }}=5500 \mathrm{~K}, \sim 0.11 \mathrm{dex}$ at $T_{\text {eff }}=5200 \mathrm{~K}$, and $\sim 0.20 \mathrm{dex}$ at $T_{\text {eff }}=4900 \mathrm{~K}$. Another weak point is the possible 0.3 dex difference between the solar photospheric Be abundance and the meteoritic Be abundance. It has been suggested that this could be due to our inability

${ }^{3}$ Here we use the notation $\log N(\mathrm{Be})=\log [N(\mathrm{Be}) / N(\mathrm{H})]+12$. 
Table 2. Stellar parameters and Be and Li abundances from the literature (García López \& Pérez de Taoro 1998; Santos et al. 2002, 2004c; Sousa et al. 2008): stars hosting planets.

\begin{tabular}{|c|c|c|c|c|c|c|c|c|c|c|}
\hline Name & $\begin{array}{l}T_{\text {eff }} \\
{[\mathrm{K}]}\end{array}$ & $\begin{array}{c}\log g \\
{\left[\mathrm{~cm} \mathrm{~s}^{-2}\right]}\end{array}$ & {$[\mathrm{Fe} / \mathrm{H}]$} & $\begin{array}{c}\text { Mass }^{a} \\
M_{\odot}\end{array}$ & $\mathrm{SpT}^{b}$ & $\mathrm{LC}^{c}$ & $\begin{array}{c}\log N(\mathrm{Be}) \\
{[\mathrm{dex}]}\end{array}$ & $\begin{array}{c}\log N(\mathrm{Li}) \\
{[\mathrm{dex}]}\end{array}$ & $\begin{array}{c}{[\mathrm{O} / \mathrm{H}]^{d}} \\
{[\mathrm{dex}]}\end{array}$ & $\begin{array}{l}\mathrm{Age}^{e} \\
\text { Gyr }\end{array}$ \\
\hline BD-103166 & 5320 & 4.38 & 0.33 & - & K0V & $\mathrm{V}$ & $<0.5$ & - & - & 5.39 \\
\hline HD 6434 & 5835 & 4.6 & -0.52 & 0.82 & G2/G3V & $\mathrm{V}$ & 1.08 & $<0.85$ & $-0.18 \pm 0.1$ & 13.3 \\
\hline HD 9826 & 6212 & 4.26 & 0.13 & 1.30 & F8V & $\mathrm{V}$ & 1.05 & 2.55 & $0.22 \pm 0.12$ & - \\
\hline HD 10647 & 6143 & 4.48 & -0.03 & 1.20 & F9V & V & 1.19 & 2.8 & $-0.06 \pm 0.09$ & 4.8 \\
\hline HD 10697 & 5641 & 4.05 & 0.14 & 1.22 & G5IV & IV & 1.31 & 1.96 & - & 1.17 \\
\hline HD 12661 & 5702 & 4.33 & 0.36 & 1.05 & K0V & V & 1.13 & $<0.98$ & - & 7.05 \\
\hline HD 13445 & 5163 & 4.52 & -0.24 & 0.70 & K1V & V & $<0.4$ & $<-0.12$ & $-0.25 \pm 0.12$ & - \\
\hline HD 16141 & 5801 & 4.22 & 0.15 & 1.06 & G5IV & V & 1.17 & 1.11 & $0.19 \pm 0.10$ & 11.2 \\
\hline HD 17051 & 6252 & 4.61 & 0.26 & 1.26 & $\mathrm{~F} 9 \mathrm{VFe}+03$ & V & 1.03 & 2.66 & $0.33 \pm 0.11$ & 1.47 \\
\hline HD 19994 & 6190 & 4.19 & 0.24 & 1.37 & F8V & V & 0.93 & 1.99 & $0.31 \pm 0.12$ & 4.7 \\
\hline HD 22049 & 5073 & 4.43 & -0.13 & 0.85 & $\mathrm{~K} 2 \mathrm{~V}$ & V & 0.77 & $<0.25$ & $-0.23 \pm 0.10$ & - \\
\hline HD 27442 & 4825 & 3.55 & 0.39 & - & K2III & III & $<0.3$ & $<-0.47$ & $0.07 \pm 0.14$ & - \\
\hline HD 38529 & 5674 & 3.94 & 0.4 & 1.60 & G4V & IV & $<-0.1$ & $<0.61$ & - & 5.09 \\
\hline HD 46375 & 5268 & 4.41 & 0.2 & 0.82 & K1IV & $\mathrm{V}$ & $<0.8$ & $<-0.02$ & $0.04 \pm 0.10$ & 16.4 \\
\hline HD 52265 & 6103 & 4.28 & 0.23 & 1.20 & G0III-IV & V & 1.25 & 2.88 & $0.21 \pm 0.10$ & 3.8 \\
\hline HD 69830 & 5410 & 4.38 & -0.03 & 0.82 & $\mathrm{~K} 0 \mathrm{~V}$ & V & 0.79 & $<0.47$ & & \\
\hline HD 75289 & 6143 & 4.42 & 0.28 & 1.24 & $\mathrm{~F} 9 \mathrm{VFe}+03$ & $\mathrm{~V}$ & 1.36 & 2.85 & $0.22 \pm 0.11$ & 4.0 \\
\hline HD $75732 \mathrm{~A}^{f}$ & 5150 & 4.15 & 0.29 & 0.87 & G8V & IV & 0.55 & $<0.04$ & - & - \\
\hline HD 82943 & 6016 & 4.46 & 0.3 & 1.15 & $\mathrm{~F} 9 \mathrm{VFe}+05$ & $\mathrm{~V}$ & 1.27 & 2.51 & $0.29 \pm 0.11$ & 3.5 \\
\hline HD 83443 & 5454 & 4.33 & 0.35 & 0.95 & $\mathrm{~K} 0 \mathrm{~V}$ & $\mathrm{~V}$ & $<0.7$ & $<0.52$ & $0.12 \pm 0.13$ & 2.94 \\
\hline HD 92788 & 5821 & 4.45 & 0.32 & 1.03 & G5 & V & 1.19 & 1.34 & - & 9.6 \\
\hline HD 95128 & 5954 & 4.44 & 0.06 & 1.07 & G1V & $\mathrm{V}$ & 1.23 & 1.83 & - & - \\
\hline HD 108147 & 6248 & 4.49 & 0.2 & 1.26 & $\mathrm{~F} 8 \mathrm{VH}+04$ & V & 0.99 & 2.33 & - & 1.98 \\
\hline HD 114762 & 5884 & 4.22 & -0.7 & 0.81 & F9V & $\mathrm{V}$ & 0.82 & 2.2 & - & 11.8 \\
\hline HD 117176 & 5560 & 4.07 & -0.06 & 0.93 & G5V & IV & 0.86 & 1.88 & - & - \\
\hline HD 120136 & 6339 & 4.19 & 0.23 & 1.33 & F6IV & $\mathrm{V}$ & $<0.25$ & - & - & - \\
\hline HD 121504 & 6075 & 4.64 & 0.16 & 1.13 & $\mathrm{G} 2 \mathrm{~V}$ & V & 1.33 & 2.65 & - & 1.62 \\
\hline HD 130322 & 5392 & 4.48 & 0.03 & 0.88 & K0III & V & 0.95 & $<0.13$ & - & 1.24 \\
\hline HD 134987 & 5776 & 4.36 & 0.3 & 1.03 & G5V & V & 1.22 & $<0.74$ & - & 11.1 \\
\hline HD 143761 & 5853 & 4.41 & -0.21 & 0.95 & G0Va & $\mathrm{V}$ & 1.11 & 1.46 & $-0.09 \pm 0.08$ & - \\
\hline HD 145675 & 5311 & 4.42 & 0.43 & 0.90 & K0V & $\mathrm{V}$ & $<0.65$ & $<0.03$ & - & 7.6 \\
\hline HD 168443 & 5617 & 4.22 & 0.06 & 0.96 & G6V & V & 1.11 & $<0.78$ & - & 10.6 \\
\hline HD 169830 & 6299 & 4.1 & 0.21 & 1.42 & F7V & $\mathrm{V}$ & $<-0.4$ & $<1.16$ & $0.22 \pm 0.12$ & 2.3 \\
\hline HD 179949 & 6260 & 4.43 & 0.22 & 1.28 & $\mathrm{~F} 8.5 \mathrm{~V}$ & $\mathrm{~V}$ & 1.08 & 2.65 & $0.26 \pm 0.11$ & 2.05 \\
\hline HD $186427^{f}$ & 5700 & 4.35 & 0.06 & 0.99 & G3V & $\mathrm{V}$ & 1.3 & 0.46 & - & - \\
\hline HD 187123 & 5845 & 4.42 & 0.13 & 1.04 & G5 & V & 1.08 & 1.21 & - & 7.3 \\
\hline HD 192263 & 4947 & 4.51 & -0.02 & 0.69 & $\mathrm{~K} 2 \mathrm{~V}$ & $\mathrm{~V}$ & $<0.9$ & $<-0.39$ & $-0.17 \pm 0.09$ & 0.57 \\
\hline HD 195019 & 5842 & 4.32 & 0.08 & 1.06 & G3IV-V & $\mathrm{V}$ & 1.15 & 1.47 & - & 10.6 \\
\hline HD 202206 & 5752 & 4.5 & 0.35 & 1.04 & G6V & $\mathrm{V}$ & 1.04 & 1.04 & $0.20 \pm 0.09$ & 2.04 \\
\hline HD 209458 & 6117 & 4.48 & 0.02 & 1.15 & G0V & $\mathrm{V}$ & 1.24 & 2.7 & $0.03 \pm 0.07$ & 6.6 \\
\hline HD 210277 & 5532 & 4.29 & 0.19 & 0.90 & G0V & V & 0.91 & $<0.3$ & $0.13 \pm 0.12$ & 6.93 \\
\hline HD 217014 & 5804 & 4.42 & 0.2 & 1.05 & G5V & V & 1.02 & 1.3 & $-0.06 \pm 0.11$ & - \\
\hline HD 217107 & 5646 & 4.31 & 0.37 & 1.02 & G8IV & V & 0.96 & $<0.4$ & $0.29 \pm 0.13$ & 7.32 \\
\hline HD 222582 & 5843 & 4.45 & 0.05 & 0.97 & G5 & V & 1.14 & $<0.59$ & $0.09 \pm 0.08$ & 11.1 \\
\hline
\end{tabular}

Notes. ${ }^{(a)}$ Values taken from Sousa et al. (2008) and Santos et al. (2004a). ${ }^{(b)}$ Values taken from SIMBAD. ${ }^{(c)}$ Luminosity class assigned by comparing the stellar parameters with isochrones from Girardi et al. (2000). ${ }^{(d)}$ Values taken from Ecuvillon et al. (2006). ${ }^{(e)}$ Values taken from Saffe et al. (2005). ${ }^{(f)}$ Values taken from García López \& Pérez de Taoro (1998).

to properly account for all continuum opacity sources in the UV (Balachandran \& Bell 1998). However, other authors have argued that Kurucz atmospheric models are able to reproduce the near-UV absolute continuum at least for stars in the $T_{\text {eff }}$ range 4000-6000 K (Allende Prieto \& Lambert 2000). Indeed, Balachandran \& Bell (1998) argue that the Fe I bound-free opacity should be increased by a factor of 1.6, which is equivalent to an increase of 0.2 dex in the Fe abundance. Smiljanic et al. (2009) have tested this possibility for a model of $[\mathrm{Fe} / \mathrm{H}]-0.5 \mathrm{dex}$ and they find that the difference in Be abundance is small, $\Delta \log N(\mathrm{Be}) \sim 0.02$ dex.

For stars with effective temperatures below $5100 \mathrm{~K}$, the spectral region surrounding the Be II $\lambda 3131 \AA$ line begins to be dominated by the contribution of other species (Mn I, C, OH lines, etc.) and for the giant stars also the thulium line (Tm II 13131.255 ^) may play an important role (see Melo et al. 2005). This makes it more difficult to fit the observed spectra and thus, to measure Be abundance. Although we took into account some of these other elements when fitting cooler and giant objects, we assume that $\mathrm{Be}$ abundances are not very accurate in these cases. In addition, the sensitivity of the Be II to the Be abundance decreases towards lower temperatures. Thus, the total error, including mainly the uncertainties on the effective temperature and the continuum location, in the $\mathrm{Be}$ abundance is of the order of $0.1 \mathrm{dex}$ at $T_{\text {eff }} \sim 6000 \mathrm{~K}, 0.12 \mathrm{dex}$ at $T_{\text {eff }} \sim 5500 \mathrm{~K}, 0.17 \mathrm{dex}$ at $T_{\text {eff }} \sim 5200 \mathrm{~K}$, and almost 0.3 dex at $T_{\text {eff }} \sim 4900 \mathrm{~K}$. 
Table 3. Stellar parameters and Be and Li abundances from literature (see García López \& Pérez de Taoro 1998; Santos et al. 2002, 2004c; Sousa et al. 2008): "Single Stars".

\begin{tabular}{|c|c|c|c|c|c|c|c|c|}
\hline Name & $\begin{array}{l}T_{\text {eff }} \\
{[\mathrm{K}]}\end{array}$ & $\begin{array}{c}\log g \\
{\left[\mathrm{~cm} \mathrm{~s}^{-2}\right]}\end{array}$ & {$[\mathrm{Fe} / \mathrm{H}]$} & $\begin{array}{c}\text { Mass }^{a} \\
M_{\odot}\end{array}$ & $\mathrm{SpT}^{b}$ & $\mathrm{LC}^{c}$ & $\begin{array}{c}\log N(\mathrm{Be}) \\
{[\mathrm{dex}]}\end{array}$ & $\begin{array}{c}\log N(\mathrm{Li}) \\
{[\mathrm{dex}]}\end{array}$ \\
\hline HD 870 & 5447 & 4.57 & -0.07 & 0.86 & K0V & $\mathrm{V}$ & 0.8 & $<0.2$ \\
\hline HD 1461 & 5768 & 4.37 & 0.17 & 1.02 & G0V & V & 1.14 & $<0.51$ \\
\hline HD 1581 & 5956 & 4.39 & -0.14 & 1.00 & F9.5V & V & 1.15 & 2.37 \\
\hline HD 3823 & 5948 & 4.06 & -0.25 & 1.01 & G0VFe-09H-04 & IV & 1.02 & 2.41 \\
\hline HD 4391 & 5878 & 4.74 & -0.03 & 1.11 & G5VFe-08 & V & 0.64 & $<1.09$ \\
\hline HD 7570 & 6140 & 4.39 & 0.18 & 1.20 & $\mathrm{~F} 9 \mathrm{VFe}+04$ & V & 1.17 & 2.91 \\
\hline HD 10700 & 5344 & 4.57 & -0.52 & 0.63 & G8V & V & 0.83 & $<0.41$ \\
\hline HD 14412 & 5368 & 4.55 & -0.47 & 0.73 & G8V & V & 0.8 & $<0.44$ \\
\hline HD 20010 & 6275 & 4.4 & -0.19 & 1.33 & F6V & V & 1.01 & 2.13 \\
\hline HD 20766 & 5733 & 4.55 & -0.21 & 0.93 & $\mathrm{G} 2 \mathrm{~V}$ & V & $<-0.09$ & $<0.97$ \\
\hline HD 20794 & 5444 & 4.47 & -0.38 & 0.70 & G8V & V & 0.91 & $<0.52$ \\
\hline HD 20807 & 5843 & 4.47 & -0.23 & 0.95 & G0V & V & 0.36 & $<1.07$ \\
\hline HD 23249 & 5074 & 3.77 & 0.13 & 0.83 & K0IV & IV & $<0.15$ & 1.24 \\
\hline HD 23484 & 5176 & 4.41 & 0.06 & 0.82 & K2Vk: & V & $<0.70$ & $<0.4$ \\
\hline HD 26965A & 5126 & 4.51 & -0.31 & 0.67 & $\mathrm{~K} 1 \mathrm{~V}$ & V & 0.76 & $<0.17$ \\
\hline HD 30495 & 5868 & 4.55 & 0.02 & 1.10 & G1.5VH-05 & V & 1.16 & 2.44 \\
\hline HD 36435 & 5479 & 4.61 & 0.00 & 0.98 & G9V & V & 0.99 & 1.67 \\
\hline HD 38858 & 5752 & 4.53 & -0.23 & 0.90 & G4V & V & 1.02 & 1.64 \\
\hline HD 43162 & 5633 & 4.48 & -0.01 & 1.00 & G6.5V & V & 1.08 & 2.34 \\
\hline HD 43834 & 5594 & 4.41 & 0.1 & 0.93 & G7V & V & 0.94 & 2.3 \\
\hline HD 72673 & 5242 & 4.5 & -0.37 & 0.70 & G9V & V & 0.7 & $<0.48$ \\
\hline HD 74576 & 5000 & 4.55 & -0.03 & 0.78 & $\mathrm{~K} 2.5 \mathrm{Vk}:$ & V & 0.7 & 1.72 \\
\hline HD 76151 & 5803 & 4.5 & 0.14 & 1.04 & $\mathrm{G} 2 \mathrm{~V}$ & V & 1.02 & 1.88 \\
\hline HD 84117 & 6167 & 4.35 & -0.03 & 1.15 & F8V & V & 1.11 & 2.64 \\
\hline HD $186408^{d}$ & 5750 & 4.2 & 0.11 & - & $\mathrm{G} 1.5 \mathrm{Vb}$ & V & 1.1 & 1.24 \\
\hline HD 189567 & 5765 & 4.52 & -0.23 & 0.87 & G2V & V & 1.06 & $<0.82$ \\
\hline HD 192310 & 5069 & 4.38 & -0.01 & 0.80 & $\mathrm{~K} 2+\mathrm{V}$ & V & $<0.6$ & $<0.2$ \\
\hline HD 211415 & 5890 & 4.51 & -0.17 & 0.93 & G0V & V & 1.12 & 1.92 \\
\hline HD 222335 & 5260 & 4.45 & -0.16 & 0.77 & G9.5V & V & 0.66 & $<0.31$ \\
\hline
\end{tabular}

Notes. ${ }^{(a)}$ Values taken from Sousa et al. (2008) and Santos et al. (2004a). ${ }^{(b)}$ Values taken from SIMBAD. ${ }^{(c)}$ Luminosity class assigned by comparing the stellar parameters with isochrones from Girardi et al. (2000). ${ }^{(d)}$ Values taken from García López \& Pérez de Taoro (1998).

All Be abundances measured in this work are listed in Table 1. We also added the Be abundance measurements of the 44 stars hosting planets and 29 "single" stars from previous studies (García López \& Pérez de Taoro 1998; Santos et al. 2002, 2004b,c), listed in Tables 2 and 3. We compiled Li abundance measurements obtained from the Li I $\lambda 6708 \AA$ line from the literature (Israelian et al. 2004, 2009). We also measured new $\mathrm{Li}$ abundances in four objects (three giants and a subgiant, see Table 1) in the same way as in Israelian et al. (2004, 2009). In Fig. 2 we display some synthetic spectral fits to the observed spectra of four stars of our sample.

\subsection{Ages}

We gathered together the ages of some of our stars hosting planets from Saffe et al. (2005). Saffe et al. (2005) studied the correlations between stellar properties with age. They measured the chromospheric activity in a sample of 49 stars with planetary companions, and combining this with the literature, they obtained age estimates for 112 objects. They applied the calibrations reported in Donahue (1993) and Rocha-Pinto \& Maciel (1998) for the chromospheric activity-age relation but also other methods based on isochrones, lithium abundances, metallicities, and kinematics, and they compared them with the chromospheric results. They concluded that chromospheric activity and isochrone methods give comparable results, and claimed that isochrone technique is, in practice, the only tool currently available to derive ages for the complete sample of planethost stars, because the chromospheric activity calibrations of Donahue (1993) are limited for ages <2 Gyr and most of the planet-host stars sample are $>2$ Gyr age. Lithium cannot be used to derive stellar ages greater than 1 Gyr. Here, we adopted the ages derived from isochrones when available for objects with age $<2$ Gyr, otherwise we used those derived using the chromospheric activity technique with the Donahue (1993) calibrations. The adopted ages are provided in Table 1 . We note that the typical uncertainty on the age determination in field stars is very large, between 1 and $3 \mathrm{Gyr}$, especially for dwarf stars.

\section{Discussion}

\subsection{Be and Li abundance versus effective temperature and mass}

In Fig. 3 we plot the derived Be abundances as a function of effective temperature (top panel) and as a function of mass (bottom panel) for the stars in our sample together with those for the stellar samples reported in García López \& Pérez de Taoro (1998); Santos et al. (2002, 2004b,c). We also distinguish among stars with and without planets and among different luminosity classes, including dwarfs (V), subgiants (IV) and giants (III). We note that we did not find any determination of the stellar mass for all stars in this study in the literature, as for instance, no stellar mass was available for subgiant and giant planet-host stars with $T_{\text {eff }}<4900 \mathrm{~K}$. 

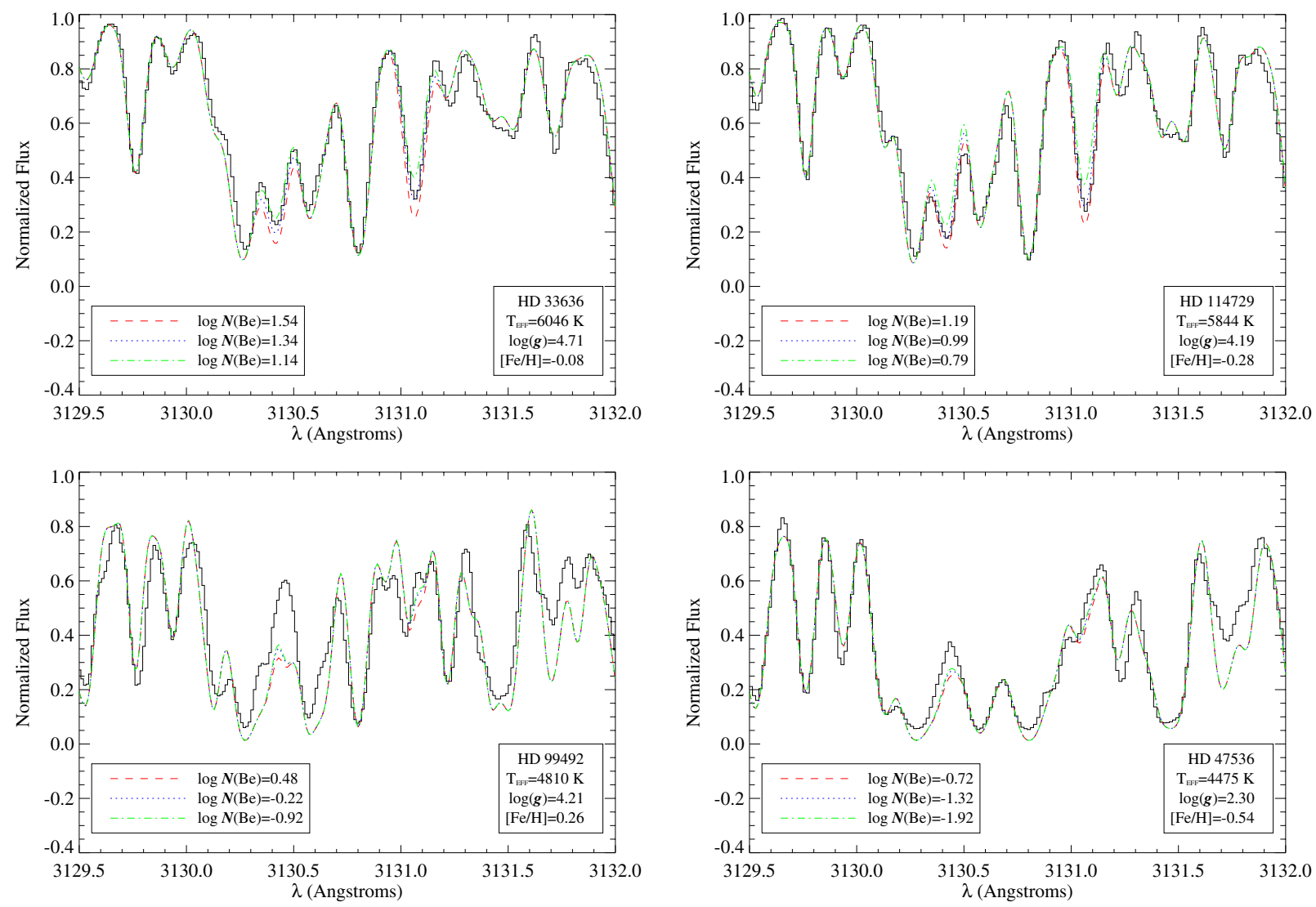

Fig. 2. Best synthetic spectral fits to the observed spectra in the Be II line region. From upper to lower from left to right, the planet-host dwarfs HD 33636 and HD 114729, the subgiant HD 99492 and the giant HD 47536 are displayed.

Subgiant and especially giant stars have probably changed their effective temperatures considerably from their "initial" value on the main sequence and their convective envelopes have also expanded and reached deeper and hotter regions in the stellar interiors. Extra mixing may have already occurred and thus, the dilution and/or depletion of their light elements do not follow the normal trend. Therefore, we will take evolved objects into account separately (see Sect. 6.1.2).

Considering only dwarfs stars, the addition of the new Be abundance of 26 planet-host stars in Fig. 3 shows no significant changes to what Santos et al. (2004c) found. The overall impression is that no clear difference seems to exist between the two stellar populations, planet hosts and "single" stars.

Be abundances decrease from a maximum near $T_{\text {eff }}=$ $6100 \mathrm{~K}$ towards higher and lower temperatures, similarly to the behaviour of $\mathrm{Li}$ abundances versus effective temperature (see Fig. 5). The steep decrease with increasing temperature resembles the well known Be gap for F stars (e.g. Boesgaard \& King 2002), while the decrease of the Be content towards lower temperatures is smoother and may show evidence for continuous Be burning during the main-sequence evolution of these stars (see Santos et al. 2004c, and references therein).

In the top panel of Fig. 3 we also posed a set of Yale beryllium-depletion isochrones from Pinsonneault et al. (1990). We depict the depletion isochrones (case A) for solar metallicity and an age of $1.7 \mathrm{Gyr}$, the standard model and four models with different initial angular momentum (see Table 3-6 of Pinsonneault et al. 1990) assuming an initial $\log N(\mathrm{Be})=1.26$ (intermediate value between solar, 1.10, and meteoritic, 1.42) for all stars (see Santos et al. 2004b, and references therein). As already noticed in Santos et al. (2004b,c), these models agree with the observations above roughly $5600 \mathrm{~K}$, but while the observed Be abundance decreases towards lower temperatures when $T_{\text {eff }}<5600 \mathrm{~K}$, these models predict either constant or increasing Be abundances. Several possibilities were also discussed for this discrepancy between models and Be observations: the increasing difficulty to measure Be abundances at low temperatures; the lack of near-UV line-opacity in the spectral synthesis; the presence of the planetary companions; possible accretion of $\approx 0.5 M_{\odot}$ by stars in the solar neighbourhood (Murray et al. 2001); and possible correlation with oxygen abundances.

All these explanations are dismissed as the main possible cause, although we will further discuss the last one in Sect. 6.4. The better explanation is probably that these models do not correctly predict the behaviour of the $\mathrm{Be}$ abundances at the lowest temperatures.

Mixing by internal gravity waves may provide an explanation to the Li and Be depletion in cool dwarf stars (García Lopez \& Spruit 1991; Montalbán 1994; Montalbán \& Schatzman 1996; Charbonnel \& Talon 2005). In Fig. 3 we also depict one model that includes mixing by gravity waves from Montalbán \& Schatzman (2000). Santos et al. (2004b) already pointed out that models including mixing by internal waves (Montalbán \& Schatzman 2000) reproduce the decrease of Be content in the lower temperature regime, although they still overestimate the Be abundances with respect to the observed values.

In the bottom panel of Fig. 3 we display the Be abundances as a function of the stellar masses. The Be abundances show, as expected, a similar trend as those of the top panel of Fig. 3 . However, some stars move with respect to the other stars in the figure, specially the evolved stars. As already mention in Sect. 6.1, the four giants and one subgiant with $T_{\mathrm{eff}}<4900 \mathrm{~K}$ 
M. C. Gálvez-Ortiz et al.: Beryllium abundances in stars with planets
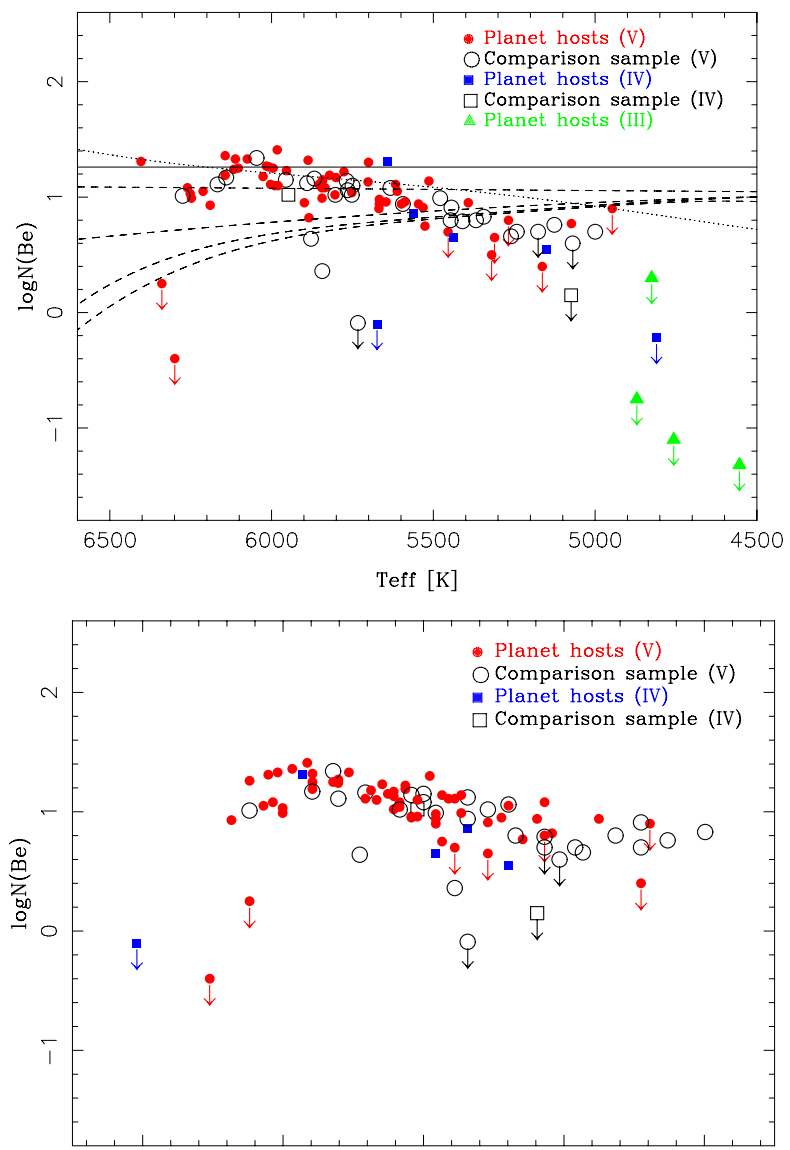

Fig. 3. Be abundances as a function of effective temperature (top panel) and as a function of mass (bottom panel) for the planet-host stars in our sample plus the stars from García López \& Pérez de Taoro (1998); Santos et al. (2002, 2004b,c). Filled and open symbols represent stars with and without planets. Circles, squares, and triangles are depicted for dwarfs (luminosity class V), subgiants (IV) and giants (III). In the top panel we superimpose the Be depletion isochrones (case A) of Pinsonneault et al. (1990) for solar metallicity and an age of $1.7 \mathrm{Gyr}$. From top to bottom, the lines represent a standard model (solid line) and four models (dashed lines) with different initial angular momentum. We also depict the Be depletion model (dotted line) including gravity waves provided in Montalbán \& Schatzman (2000).

do not have available mass determinations. A priori, one would expect to find the subgiant stars at high masses, but this picture changes for stars with different metallicities. Some subgiant stars with planets stay with relatively low masses because of their low metal content, whereas other subgiants, like HD 38529 with a mass of $1.6 M_{\odot}$ and $[\mathrm{Fe} / \mathrm{H}]=0.4$, and with an upper-limit Be measurement, have very high masses partially because of their high metallicity. Below, we will concentrate on the Be abundances in main-sequence stars.

\subsubsection{Main-sequence stars}

In the top panel of Fig. 4 we display Be abundances as a function of effective temperature only for unevolved stars with and without a known planetary companion. Here we excluded the stars with Be abundances below 0.4 dex that lie in some kind of Be-gap (see Santos et al. 2004b). We performed a linear fit, also overplotted in Fig. 4, and obtained a correlation coefficient of 0.74 with standard deviation of 0.15 . If one distinguishes between the planet hosts and the comparison sample dwarf stars, no significant difference in slope is found when fitting all stars in the whole temperature range.
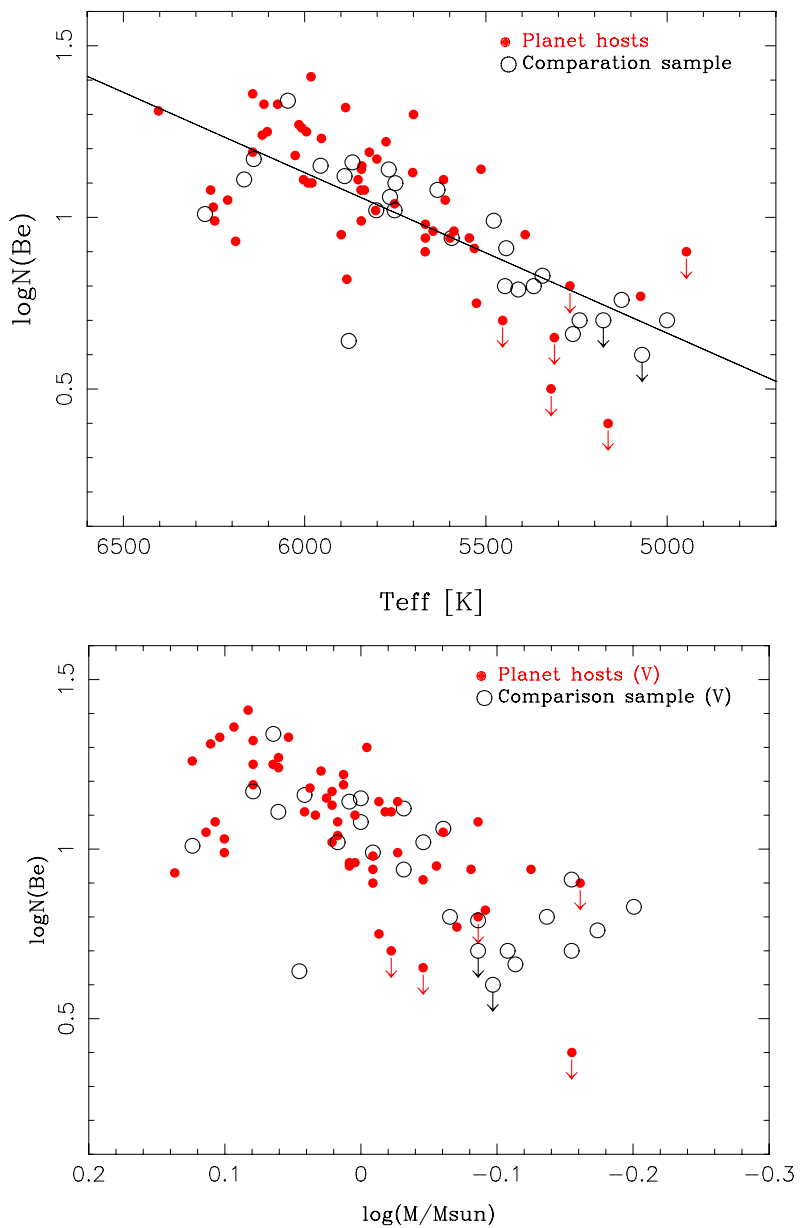

Fig. 4. Be abundances as a function of effective temperature (top panel) and as a function of mass (bottom panel) for only dwarfs with (filled circles) and without known planetary companion (open circles). Overplotted are the best linear fits found for both populations together.

In Fig. 4 (top panel) one can see that whereas in the range $5700 \lesssim T_{\text {eff }} \lesssim 6200 \mathrm{~K}$, the stars with and without planets mostly show similar abundances, at the temperature range $5100 \lesssim T_{\text {eff }} \lesssim$ $5500 \mathrm{~K}$ there seems to be some indication that planet-host stars may be more Be depleted than "single" stars.

Israelian et al. (2009), based on the Li study of an unbiased sample of solar-analogue stars with and without detected planets, find that planet hosts have lower Li abundances, being most of them upper-limit measurements, than "single" stars, which suggests that the presence of planets may increase the amount of mixing and deepen the convective zone to such an extent that the Li can be burned.

If this is true, we should see the same effect at lower temperatures in the Be burning regime. This may be reflected in the apparent overabundance of Be in "single" stars with respect to planet host stars at $5100-5500 \mathrm{~K} T_{\text {eff }}$ range, in the sense that most of the Be measurements in planet hosts are upper limits.

However, the sample with $T_{\text {eff }}$ below $5500 \mathrm{~K}$ is still too small. In addition, as shown in Sect. 5, the expected error on the Be abundances in this $T_{\text {eff }}$ range is $0.12-0.17$ dex. Therefore, this calls for new observations to add more Be measurements in dwarf stars at these cool temperatures.

In Fig. 4 (bottom panel) one can see that many points have moved to a different relative position according to the effective temperature due to the different metallicity of the stars with and without planets. In particular, the stars without planets, which 
on average have lower metallicity, appear to have lower masses. Accordingly, the points of the previous picture, which were well concentrated in a relatively narrow $T_{\text {eff }}$ range are now spread across a comparatively larger mass range. In particular, the mainsequence stars with planets in the $T_{\text {eff }}$ range $5100-5500 \mathrm{~K}$ have a mean metallicity of $[\mathrm{Fe} / \mathrm{H}] \sim 0.18$ (with a $\sigma_{\mathrm{Fe}}=0.22$ ), whereas "single" stars have a mean $[\mathrm{Fe} / \mathrm{H}] \sim-0.22\left(\sigma_{\mathrm{Fe}}=0.20\right)$. This explains why the slight signature in stars with and without planets seen in the $T_{\text {eff }}$ plot are smudged in the $\log \left(M / M_{\odot}\right)$ plot. We note that the star BD-103166, at $T_{\text {eff }} \sim 5320 \mathrm{~K}$ and with $\log N(\mathrm{Be})<0.5$, is not displayed in Figs. 3 and 4. We did not find any mass determination for this star, but we may estimate its mass to be roughly $0.91 M_{\odot}$, and thus having $\log \left(M / M_{\odot}\right) \sim$ -0.04 .

On the other hand, the stellar masses trace the stellar positions at the beginning of the stellar life and not at their present states, which are established by the stellar temperatures. As we notice in Sect. 6.3, the stellar metallicity may have an impact on the Be burning rate but we do not expect that this explains why most of the Be measurements at the $T_{\text {eff }}$ range $5100-5500 \mathrm{~K}$ in planet hosts are only upper-limits. Unfortunately, we cannot track the effect of metallicity in the pre-main sequence and mainsequence evolution of Be abundances from theoretical models at different metallicities because most of the standard models do not predict any Be depletion at these cool temperatures irrespective of the metal content of the star (see e.g. Siess et al. 2000).

In Fig. 5 we display the Li abundances of the stars in the sample versus effective temperature (top panel) and versus mass (bottom panel). All stars in this plot follow the general trend: stars with low Be abundances have their Li severely depleted as well. This general trend seen in the top panel of Fig. 5 is less clear in the bottom panel because of the larger spread of the $\mathrm{Li}$ abundance measurements in the $\log \left(M / M_{\odot}\right)$ axis. However, there is a small group of both stars with and without planets at temperatures $T_{\text {eff }} \lesssim 5670 \mathrm{~K}$ that show relatively high values of $\mathrm{Li}$ abundance, $\log N(\mathrm{Li})>1.2$, whereas their Be abundances are close to the solar value, $\log N(\mathrm{Be}) \sim 1.1$. Two of these stars are dwarf planet-host stars: HD 1237, with a relatively high chromospheric activity index, $\log R_{\mathrm{HK}}=-4.496$ (Gray et al. 2006), classified as an active star, and maybe a young object, with an age below $1 \mathrm{Gyr}$; and HD 65216, with a slightly high Li abundance, $\log N(\mathrm{Li})=1.28$, and a low chromospheric index, $\log R_{\mathrm{HK}}=-4.92$. We note that the Sun has $\log R_{\mathrm{HK}} \sim-4.75$. Among these stars there are also four "single" dwarf stars: three of them, HD 36435, HD 43162, and HD 74576, with also high chromospheric activity indices, $\log R_{\mathrm{HK}}=-4.499,-4.480$ and -4.402 , respectively. However, the other object, HD 43834 , shows a relatively low activity $\left(\log R_{\mathrm{HK}}=-4.940\right)$. This star, with a $T_{\text {eff }}=5594 \mathrm{~K}$, has a "normal" Be abundance, $\log N(\mathrm{Be})=$ 0.94 dex and a high Li abundance, $\log N(\mathrm{Li})=2.30 \mathrm{dex}$, for its temperature, according to the general trend. This makes this star interesting in the context of pollution. We refer here to the studies by Santos et al. (2004a,b) where these four "single" stars have already been discussed.

It is also worth remarking on one planet-host dwarf star of our sample, HD 142, with a temperature $T_{\text {eff }} \approx 6400 \mathrm{~K}$, with a higher Be content than expected from the typical trend, which is nearly as high as the maximum at $T_{\text {eff }} \sim 6100 \mathrm{~K}$. This star also has a higher $\mathrm{Li}$ abundance than expected from its effective temperature (see Fig. 5). We also note that the Be abundance maximum may need to be slightly shifted towards lower temperatures by one of the planet-host stars presented in this work, HD 213240, which is located at $T_{\text {eff }}=5982 \mathrm{~K}$ and has $\log N(\mathrm{Be}) \sim 1.4$. In other words, one would have to say that
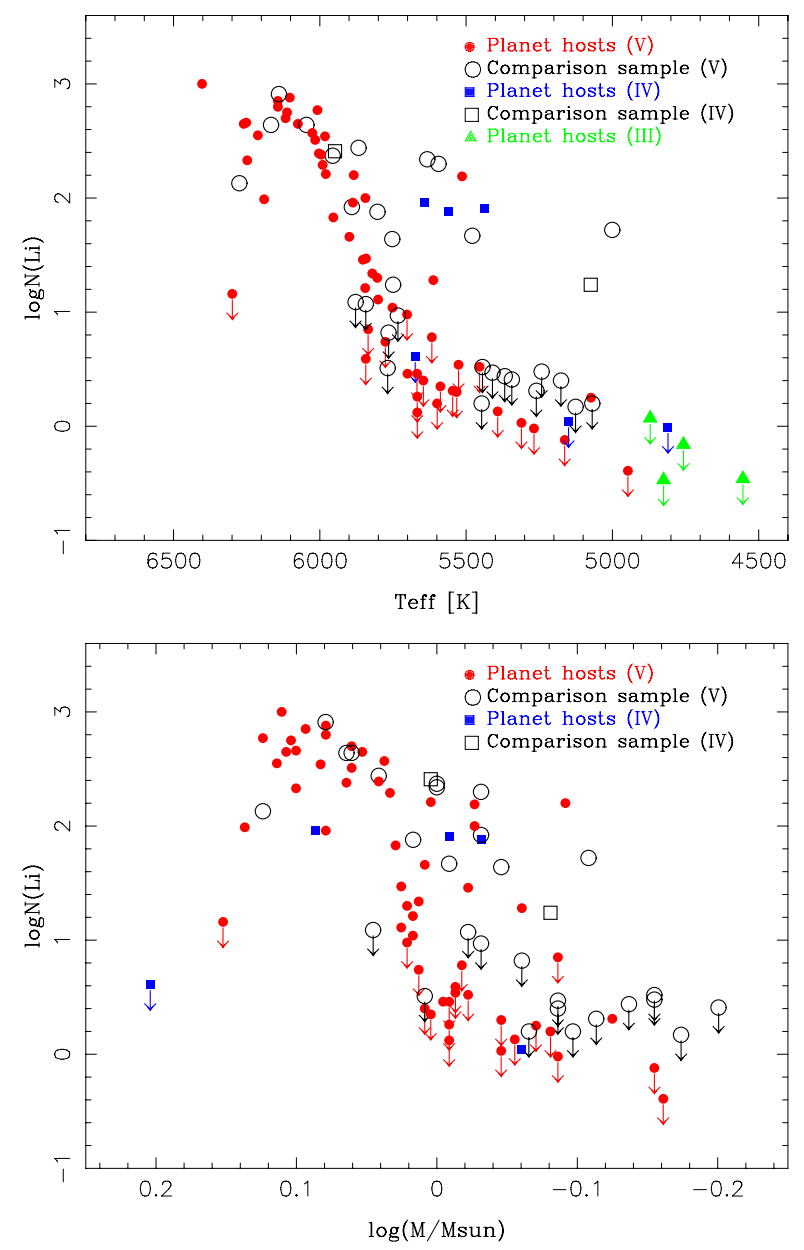

Fig. 5. Same as Fig. 3 but for Li abundance measurements taken from Israelian et al. (2004, 2009).

stars with maximum $\mathrm{Be}$ abundances have temperatures in the range $5950 \lesssim T_{\text {eff }} \lesssim 6150 \mathrm{~K}$.

The simplest explanation may be that HD 142, classified as F7V by SIMBAD ${ }^{4}$, is just a young star, which may be supported by its derived and relatively low surface gravity, $\log g=4.62$. However, according to the age estimates in Eggenberger et al. (2007), this star has an age of $\approx 2.8-5.93 \mathrm{Gyr}$, which still makes the lithium content too high. In addition, this star has a relatively low chromospheric activity index $\log R_{\mathrm{HK}}=-4.853$ (Gray et al. 2006), which may indicate that, indeed, the age determined by Eggenberger et al. (2007) may be correct. Gray et al. (2006) classified HD 142 as a chromospherically inactive star. HD 142 lies in the so-called Be "gap" according to its derived effective temperature of $T_{\text {eff }} \sim 6400 \mathrm{~K}$, but has kept nearly all its Be. In the literature one can find stars with similar or even higher Be content but typically at $T_{\text {eff }} \lesssim 6350 \mathrm{~K}$, for instance, in the Hyades cluster with an age of $600 \mathrm{Myr}$ (Boesgaard \& King 2002) and in the intermediate-age open cluster IC 4651 (Smiljanic et al. 2010) with an age of $1.7 \mathrm{Gyr}$, both with a metallicity of $\sim 0.1$ dex, very similar to the metallicity of HD 142. We note, however, that the mass of HD 142, $1.29 M_{\odot}$, places this star very close to other main-sequence stars in the bottom panel of Figs. 3 and 4.

New observations of Be in stars with and without planets are also needed in this temperature range to study possible pollution effects. We note that the depletion rates may be different

\footnotetext{
4 The SIMBAD database can be accessed at http://simbad. u-strasbg.fr/simbad/
} 
for different proto-planetary disc masses and composition, and therefore, the rotational history of the star (e.g. Balachandran 1995; Chen et al. 2001). Thus we will need to find targets with similar conditions, i.e. temperature, age, etc.

\subsubsection{Evolved stars}

Following the criterion established in Sect. 3, four objects are clearly classified as giants and eight could also have evolved off the main sequence.

Five of these subgiants have $\mathrm{Li}$ abundance measurements and four of them (the planet hosts HD 10697, HD 88133 and HD 117176 and the "single" star HD 23249) show relatively high values with respect to the Li trend described by most of the dwarf stars. However, these four subgiant stars have Be abundances consistent with the Be trend shown in dwarf stars (see Fig. 3).

HD 10697 and HD 117176, as well as the "single" star HD 23249 were already discussed in Santos et al. (2002, 2004c). These authors discussed the possibility that pollution by invoking planet engulfment (see e.g. Israelian et al. 2001, 2003), at least for the two planet hosts, could explain the high Li content and the relative "normal" Be content (Siess \& Livio 1999). However, recent models show that many accretion events of planetary material could cause Li depletion instead of Li enhancement (see e.g. Théado et al. 2010; Baraffe \& Chabrier 2010).

The high Li content and also moderate Be in the metal-rich planet host $\mathrm{HD} 88133,[\mathrm{Fe} / \mathrm{H}]=0.33$, may also be explained by pollution effects.

The four giant stars, HD 47536, HD 59686, HD 219449, and HD 27442, are all planet hosts and only present upper limits for $\mathrm{Li}$ and $\mathrm{Be}$.

\subsection{Beryllium versus lithium}

A beryllium versus lithium diagram can provide information on their different depletion rates in main-sequence stars. We already introduced the relationship between $\mathrm{Be}$ and $\mathrm{Li}$ abundance in the previous Sect. 6.1, but here, we will focus in Fig. 6, which depicts the Be abundances versus $\mathrm{Li}$ abundances of the stars in our sample. In general, there seems to be an almost constant relation between the $\mathrm{Be}$ and $\mathrm{Li}$ abundances in dwarf stars, except for very few dwarfs in the comparison sample. However, stars both with and without planets seem to follow the same behaviour.

In Fig. 6 we also split the Be and Li measurements into several stellar temperature ranges. Thus, the objects with $T_{\text {eff }} \gtrsim$ $6000 \mathrm{~K}$ are situated in the upper right corner, define the abundance maximum for both $\mathrm{Be}$ and $\mathrm{Li}$, and follow a positive correlation. The stars with temperatures between 5600 and $6000 \mathrm{~K}$ are situated in the middle of the diagram, in a nearly flat Be abundance through $\mathrm{Li}$ abundances ranging from 0.05 to $2.5 \mathrm{dex}$. The objects with temperatures between 5600 and $5100 \mathrm{~K}$ are mostly in the left upper-middle panel also follow an almost constant Be abundance at $\log N(\mathrm{Be}) \sim 0.9$ with only upper-limit Li measurements. This temperature range involves all those stars where Be depletion has already taken place and $\mathrm{Li}$ is therefore severely depleted. Thus, this group shows a Be level lower than that higher temperatures. Nevertheless, five objects in this temperature regime (two of them are subgiants), both with and without planetary companions, are located in the right upper side, and both show high levels of Be and Li abundances. Finally, we separated the objects with temperatures below $5100 \mathrm{~K}$ because the Be abundances cannot be determined accurately. These stars can

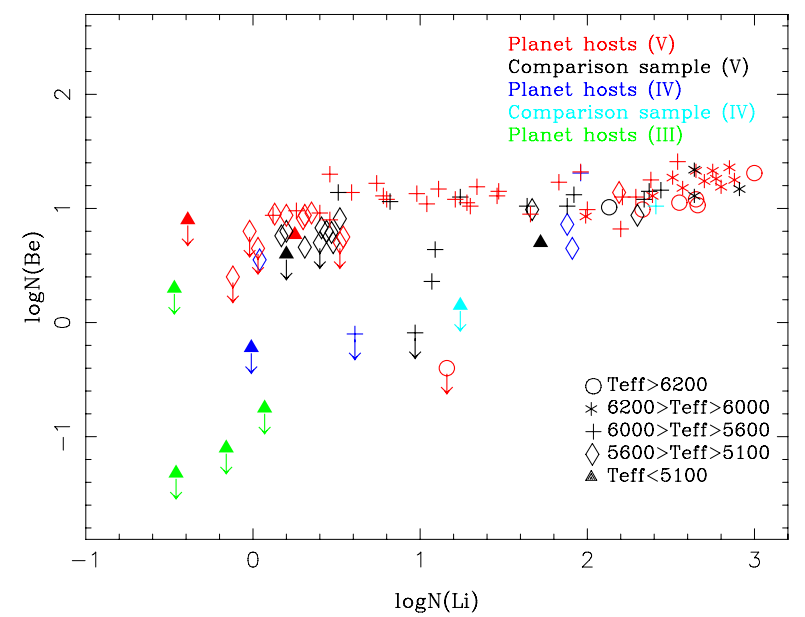

Fig. 6. Be versus $\mathrm{Li}$ abundances of the stars in the sample with and without planets. Different colours represent different luminosity classes. The stars are splitted into different temperature ranges associated with different symbols.

in turn be divided into three sets: (i) low Be and $\mathrm{Li}$ abundances that correspond to the giant stars; (ii) low Li abundances but still relatively high Be abundances; and (iii) intermediate abundances of both $\mathrm{Li}$ and $\mathrm{Be}$. The latter contains two objects, one of them is a subgiant (see Sect. 6.1.2).

Figure 6 confirms what was found in Santos et al. (2004c), $\mathrm{Be}$ and Li burning seems to follow the same trend for the hottest and coolest objects. $\mathrm{Li}$ and Be are depleted in a similar way up to $5600 \mathrm{~K}$, where the Be burning stops while the Li burning continues up to $\sim 6000 \mathrm{~K}$, where again both $\mathrm{Li}$ and Be are destroyed in a similar way. Li burning starts to be severe from $T_{\text {eff }} \lesssim 5900 \mathrm{~K}$ while Be keeps its low-burning rate until $T_{\text {eff }} \sim 5600 \mathrm{~K}$. Giant stars show both Li and Be severely depleted with exception of one object, HD 27442, which still keeps a relatively high upper limit to its Be abundance.

In general, $\mathrm{Li}$ is more depleted than $\mathrm{Be}$ in stars with lower effective temperatures and all stars with and without planetary companions show a similar behaviour. With the addition of the 26 planet-host stars and one "single" star, the apparent "lack" of planet-host stars in the range of Li abundances $\log N(\mathrm{Li}) \sim$ 1.5-2.5, noted by Santos et al. (2004c), has almost disappeared. There seems to be roughly the same number of stars with and without planets in this range of $\mathrm{Li}$ abundances, but this might also be because of the small number of stars without planets, 30, in this sample, compared with the 70 planet-host stars.

\subsection{Be abundance versus $[\mathrm{Fe} / \mathrm{H}]$ and age}

In Fig. 7 we display the Be abundances versus the metal content of the stars with and without planets. We do not see any clear correlation but just an increasing dispersion of the Be content towards higher metallicities. The differences in the Be content are mainly caused by the differences in $T_{\text {eff }}$, which are lower at lower temperatures, but roughly constant for all metallicities. This was already noticed by Santos et al. (2004c).

At the highest metallicities, the opacity of the convective zone changes considerably, producing a higher rate of Li burning and also, although less significant, Be burning. This might explain why the Be abundance in planet-host stars has a maximum at $[\mathrm{Fe} / \mathrm{H}] \sim 0.20$ and then decreases towards higher metallicities. On the other hand, Galactic Be abundances are known to increase with the metallicity (e.g. Rebolo et al. 1988; Molaro et al. 1997; Boesgaard et al. 1999), which can be seen in 


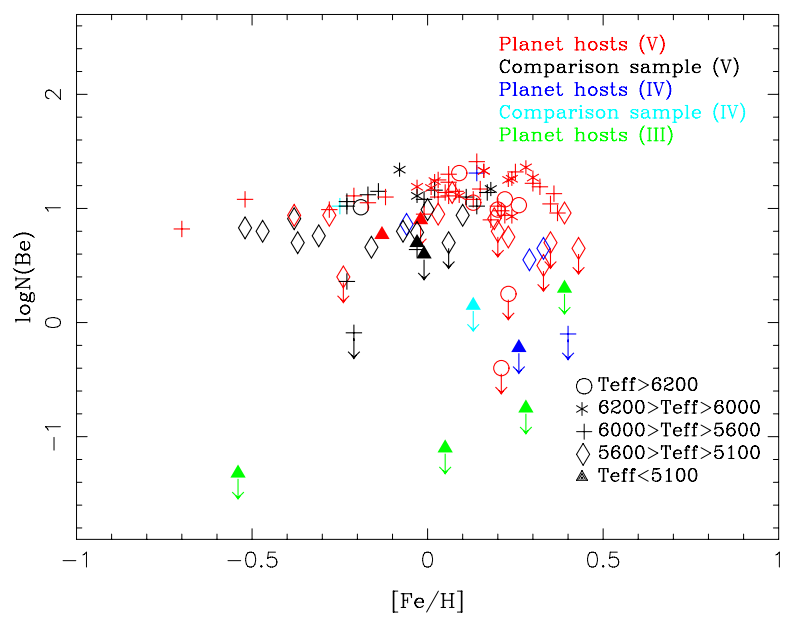

Fig. 7. Same as Fig. 6 but for Be abundance versus metallicity.

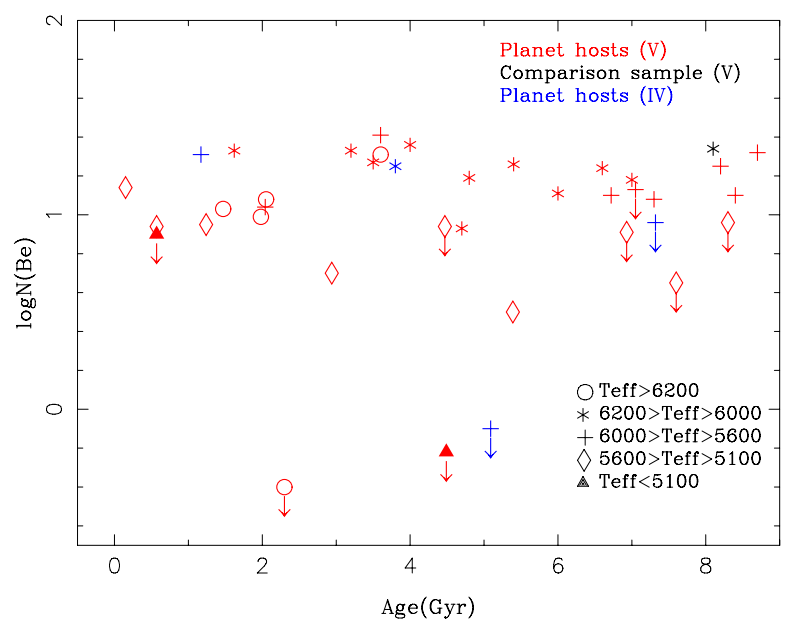

Fig. 8. Same as Fig. 6 but for Be abundance versus age.

Fig. 7, although the dispersion in Be abundances and the effective temperature spread is probably masking this Galactic effect (see also Santos et al. 2004c, and references therein).

In Fig. 8 we display the Be abundances versus ages of the planet-host stars including those from the literature. We have objects in the same temperature regime but with different ages. There is no correlation, indicating that the age as well as the metallicity is a secondary effect on the level of the Be content behind a primary effect driven by the effective temperature of the star. However, any interpretation from this plot must be taken with caution since the age determinations, specially for mainsequence stars, have large error bars and may not be reliable.

\subsection{Be versus $[\mathrm{O} / \mathrm{H}]$}

Beryllium is mainly produced in the spallation of CNO nuclei (see e.g. Tan et al. 2009, and references therein). In particular, oxygen gives the largest contribution to the spallation process responsible for forming $\mathrm{Be}$. Thus, a comparison between $\mathrm{O}$ and $\mathrm{Be}$ abundances can provide alternative information on the behaviour of beryllium. We collected the oxygen abundances in stars hosting planets of our sample from Ecuvillon et al. (2006). They found average oxygen abundances, $[\mathrm{O} / \mathrm{H}], 0.1-0.2$ dex higher in stars hosting planets with respect to stars without planets. However, although planet accretion was not excluded, the possibility that it is the main source of the observed oxygen enhancement in planet-host stars is unlikely. Thus, it seems to be related
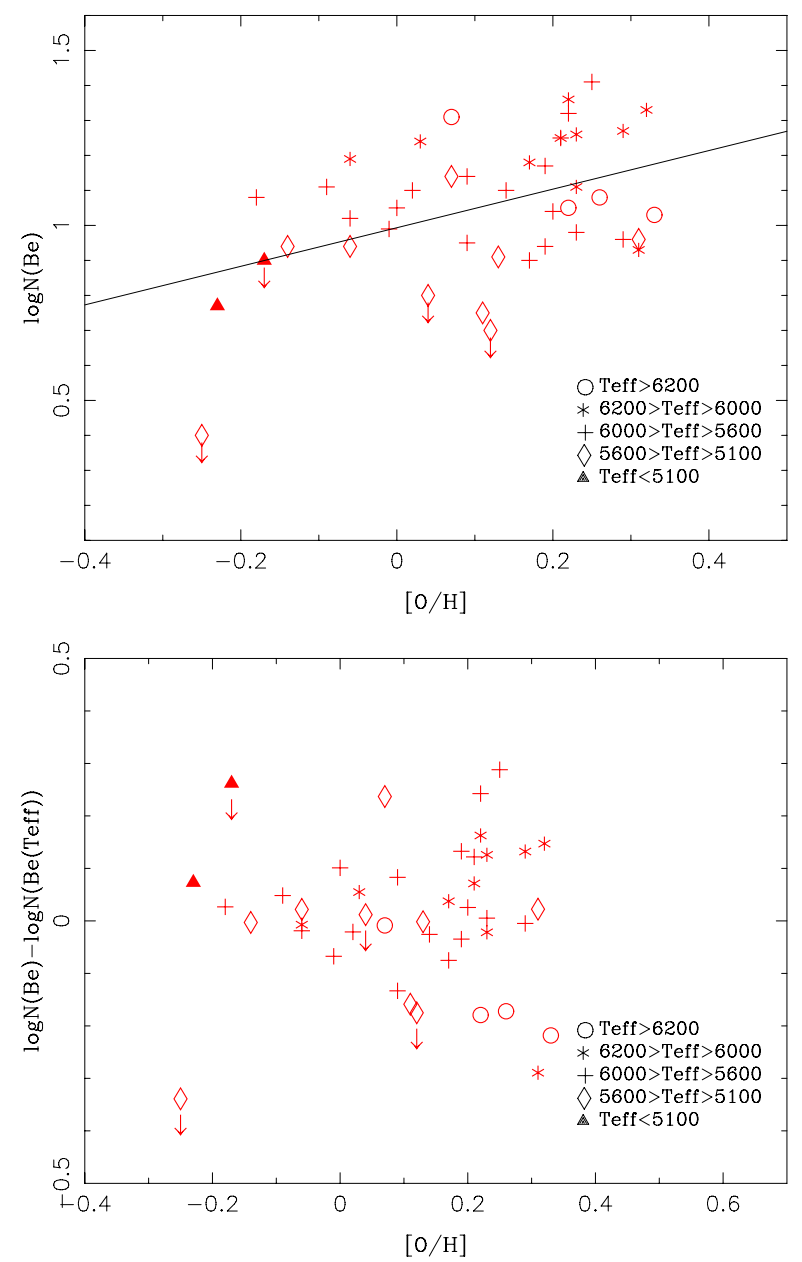

Fig. 9. Top: Be versus $[\mathrm{O} / \mathrm{H}]$ abundances in dwarf stars hosting planets in the sample and the best linear fit. Bottom: Be abundance corrected using the linear relation $\mathrm{Be}-T_{\text {eff }}$.

to the higher metal content of planet-harbouring stars and the Galactic trends of stars with and without planet are nearly indistinguishable.

In (top panel) of Fig. 9 we display the beryllium versus oxygen abundances relative to the Sun only in dwarf planet-host stars. We took the $[\mathrm{O} / \mathrm{H}]$ values derived from the near-UV OH bands in the spectral range 3167-3255 $\AA$ (Ecuvillon et al. 2006), because these lines are located close to the Be II $3131 \AA$ line and therefore would be affected for the same continuum opacity. We note that the stellar parameters used in this work to derive Be abundances are not exactly the same as those used by Ecuvillon et al. (2006) to derive oxygen abundances, but in general the differences are very small. We adopted the mean value derived from the four $\mathrm{OH}$ features as given in Ecuvillon et al. (2006). We also note that there is no available $\mathrm{O}$ measurement for some stars in Fig. 3.

The Be content is stratified in different values as the temperature changes. In the temperature range $5100-5600 \mathrm{~K}$ the $\mathrm{Be}$ abundance versus $[\mathrm{O} / \mathrm{H}]$ seems to have a positive slope, which is due to just one dwarf star located at $[\mathrm{O} / \mathrm{H}] \sim-0.25$. However, all these trends are affected by the strong influence of the effective temperature on the Be abundances in each star, irrespective of the metallicity and oxygen abundance.

In the top panel of Fig. 9, similarly to Fig. 4, we also displayed the best linear fit, showing a correlation of 0.44 with a standard deviation of 0.18 . To study the influence of the effective 
temperature in this $\mathrm{Be}-\mathrm{O}$ relation, we subtract from each $\mathrm{Be}$ measurement the $\mathrm{Be}$ content given by the $\mathrm{Be}-T_{\text {eff }}$ linear relation in Fig. 4. We thus obtain the Be abundance "corrected" from the effect of the effective temperature, $\log N(\mathrm{Be})-\log N\left(\mathrm{Be}\left(T_{\mathrm{eff}}\right)\right)$, which is shown in the bottom panel of Fig. 9. The Be content does not seem to depend much on the initial oxygen abundance of the star, because the trend disappears when removing the $T_{\text {eff }}$ effect.

\section{Summary and conclusions}

We present here new Be abundances of 26 stars that harbour planets and one star without detected planets from unpublished UVES spectra, added to the previous sample of García López \& Pérez de Taoro (1998); Santos et al. (2002, 2004b,c). We also compiled Li abundances for these objects. The complete sample contains 100 objects, including dwarfs, subgiants and giants, with 70 stars hosting planets. We studied the behaviour of $\mathrm{Be}$ abundances of the stars in the sample with effective temperature, Li abundance, metallicity, age, oxygen abundance and through different spectral types and luminosity classes.

In general, we conclude that the beryllium content in stars with and without planetary companion is similar and behaves in the same way. Because the burning process is sensitive to the mixing mechanism, the presence of a planetary companion is not necessary affecting this process in a notorious way, at least, at $T_{\text {eff }} \gtrsim 5500 \mathrm{~K}$.

Below $T_{\text {eff }} \approx 5500 \mathrm{~K}$, the stellar sample is still small but there appears to be an increase in the dispersion of the Be abundances of dwarf stars. In addition, most of the planet-host Be measurements are indeed not measurements but upper limits. However, there is no way to check if the apparent higher scatter of Be content at $T_{\text {eff }} \lesssim 5500 \mathrm{~K}$ is caused by the different stellar metallicities because most of the standard theoretical models predict no Be depletion at these temperatures for any metallicity.

Be depletion depends on the effective temperature more than on the age and metal content. The fact that the stars which host planets are richer in different metal contents than "single" stars, but have the same Be content, supports the idea of the primordial origin of these over-abundances in planet hosts.

Acknowledgements. M.C.G.O. acknowledges financial support from the European Commission in the form of a Marie Curie Intra European Fellowship (PIEF-GA-2008-220679) and the partial support by the Spanish MICINN under the Consolider-Ingenio 2010 Program grant CSD2006-00070: First Science with the GTC (http://www.iac.es/consolider-ingenio-gtc). N.C.S. would like to acknowledge the support by the European Research Council/European Community under the FP7 through a Starting Grant, as well from Fundação para a Ciência e a Tecnologia (FCT), Portugal, through a Ciência 2007 contract funded by FCT/MCTES (Portugal) and POPH/FSE (EC), and in the form of grant reference PTDC/CTE-AST/098528/2008 from FCT/MCTES. J.I.G.H. is grateful for the financial support from the Spanish Ministry project MICINN AYA2008-00695. E.D.M and G.I. acknowledge the financial support from the Spanish Ministry project MICINN AYA 2008-04874. This research has made use of the SIMBAD database, operated at CDS, Strasbourg, France. This work has also made use of the IRAF facility.

\section{References}

Allende Prieto, C., \& Lambert, D. L. 2000, AJ, 119, 2445

Allende Prieto, C., Barklem, P. S., Lambert, D. L., \& Cunha, K. 2004, A\&A, 420,183
Ashwell, J. F., Jeffries, R. D., Smalley, B., et al. 2005, MNRAS, 363, L81

Balachandran, S. C. 1995, AJ, 446, 203

Balachandran, S. C., \& Bell, R. A. 1998, Nature, 392, 791

Baraffe, I., \& Chabrier, G. 2010, A\&A, 521, A44

Bertelli, G., Girardi, L., Marigo, P., \& Nasi, E. 2008, A\&A, 484, 815

Boesgaard, A. M., \& King, J. R. 2002, ApJ, 565, 587

Boesgaard, A. M., Deliyannis, C. P., King, J. R., et al. 1999, AJ, 117, 1549

Bouvier, J. 2008, A\&A, 489, L53

Charbonnel, C., \& Talon, S. 2005, Science, 309, 2189

Chen, Y. Q., \& Zhao, G. 2006, AJ, 131, 1816

Chen, Y. Q., Nissen, P. E., Benon, T., \& Zhao, G. 2001, A\&A, 371, 943

Donahue, R. A. 1993, Ph.D. Thesis, New Mexico State University

Ecuvillon, A., Israelian, G., Santos, N. C., et al. 2004a, A\&A, 418, 703

Ecuvillon, A., Israelian, G., Santos, N. C., et al. 2004b, A\&A, 426, 619

Ecuvillon, A., Israelian, G., Santos, N. C., et al. 2006, A\&A, 445, 633

Eggenberger, A., Udry, S., Chauvin, G., et al. 2007, A\&A, 474, 273

Fischer, D. A., \& Valenti, J. 2005, ApJ, 622, 1102

García López, R. J., \& Spruit, H. C. 1991, ApJ, 377, 268

García López, R. J., \& Pérez de Taoro, M. R. 1998, A\&A, 334, 599

García López, R. J., Rebolo, R., \& Martín, E. L. 1994, A\&A, 282, 518

Gilli, G., Israelian, G., Ecuvillon, A., Santos, N. C., \& Mayor, M. 2006, A\&A, 449, 723

Girardi, L., Bressan, A., Bertelli, G., \& Chiosi, C. 2000, A\&AS, 141, 371 G

Gonzalez, G. 2008, MNRAS, 386, 928

Gonzalez, G., Laws, C., Tyagi, S., \& Reddy, B. E. 2001, AJ, 121, 432

Gray, D. F. 1992, Camb. Astrophys. Ser., 20

Gray, R. O., Corbally, C. J., Garrison, R. F., et al. 2006, AJ, 132, 161

Haywood, M. 2009, ApJ, 698, L1

Israelian, G., Santos, N. C., Mayor, M., \& Rebolo, R. 2001, Nature, 411, 163 Israelian, G., Santos, N. C., Mayor, M., \& Rebolo, R. 2003, A\&A, 405, 753

Israelian, G., Santos, N. C., Mayor, M., \& Rebolo, R. 2004, A\&A, 414, 601

Israelian, G., Delgado Mena, E., Santos, N. C., et al. 2009, Nature, 462, 189

Kurucz, R. 1993, ATLAS9 Stellar Atmosphere Programs and 2 km/s grid,

Kurucz CD-ROM No. 13, Cambridge, Mass.: Smithsonian Astrophysical Observatory, 13

Laws, C., Gonzalez, G., Walker, K. M., et al. 2003, AJ, 125, 2664

Melo, C. H. F., de Laverny, P., Santos, N. C., et al. 2005, A\&A, 439, 227

Molaro, P., Bonifacio, P., Castelli, F., \& Pasquini, L. 1997, A\&A, 319, 593

Montalbán, J. 1994, A\&A, 281, 421

Montalbán, J., \& Schatzman, E. 1996, A\&A, 305, 513

Montalbán, J., \& Schatzman, E. 2000, A\&A, 354, 943

Murray, N., Chaboyer, B., Arras, P., Hansen, B., \& Noyes, R. W. 2001, ApJ, 555 , 801

Neves, V., Santos, N. C., Sousa, S. G., Correia, A. C. M., \& Israelian, G. 2009, A\&A, 497, 563

Pasquini, L., Döllinger, M. P., Weiss, A., et al. 2007, A\&A, 473, 979

Pinsonneault, M. H., Kawaler, S. D., \& Demarque, P. 1990, ApJS, 74, 501

Randich, S., Aharpour, N., Pallavicini, R., Prosser, C. F., \& Stauffer, J. R. 1997, A\&A, 323, 86

Randich, S., Primas, F., Pasquini, L., \& Pallavicini, R. 2002, A\&A, 387, 222

Rebolo, R., Abia, C., Beckman, J. E., \& Molaro, P. 1988, A\&A, 193, 193

Rocha-Pinto, H.-J., \& Maciel, W.-J. 1998, MNRAS, 298, 332

Saffe, C., Gómez, M., \& Chavero, C. 2005, A\&A, 443, 609

Santos, N. C., Israelian, G., \& Mayor, M. 2001, A\&A, 373, 1019

Santos, N. C., García López, R. J., Israelian, G., et al. 2002, A\&A, 386, 1028

Santos, N. C., Israelian, G., Mayor, M., Rebolo, R., \& Udry, S. 2003, A\&A, 398, 363

Santos, N. C., Israelian, G., \& Mayor, M. 2004a, A\&A, 415, 1153

Santos, N. C., Israelian, G., Randich, S., García López, R. J., \& Rebolo, R. 2004b, A\&A, 425, 1013

Santos, N. C., Israelian, G., García López, R. J., et al. 2004c, A\&A, 427, 1085

Santos, N. C., Israelian, G., Mayor, M., et al. 2005, A\&A, 437, 1127

Siess, L., \& Livio, M. 1999, MNRAS, 308, 1133

Siess, L., Dufour, E., \& Forestini, M. 2000, A\&A, 358, 593

Smiljanic, R., Pasquini, L., Bonifacio, P., et al. 2009, A\&A, 499, 103

Smiljanic, R., Pasquini, L., Charbonnel, C., \& Lagarde, N. 2010, A\&A, 510, A50

Sneden, C. 1973, Ph.D. Dissertation, Univ. of Texas, Austin

Sousa, S. G., Santos, N. C., Mayor, M., et al. 2008, A\&A, 487, 373

Takeda, Y., Kawanomoto, S., Honda, S., Ando, H., \& Sakurai, T. 2007, A\&A, 468,663

Tan, K. F., Shi, J. R., \& Zhao, G. 2009, MNRAS, 392, 205

Théado, S., Bohuon, E., \& Vauclair, S. 2010, IAU Symp., 268, 427 